\title{
Bioinformatics Study on Relationship of Hyperlipidemia and Phospholipids Based on Cytoscape Software
}

\author{
Zhongfeng Shi \\ Central Laboratory \\ GuangDong Pharmaceutical University \\ Guangzhou, China \\ e-mail: shizhf@139.com \\ Yifan Feng \\ Central Laboratory \\ GuangDong Pharmaceutical University \\ Guangzhou, China \\ e-mail: yiffeng@139.com
}

\author{
Wen Rui \\ Central Laboratory \\ GuangDong Pharmaceutical University \\ Guangzhou, China \\ e-mail: r_wing@139.com \\ Jiao Guo* \\ Institute of traditional Chinese Medicine \\ GuangDong Pharmaceutical University \\ Guangzhou, China \\ e-mail: guoj@gdpu.edu.cn (corresponding author)
}

\begin{abstract}
Objective : Phospholipids are one of important factors in Hyperlipidemia development. To discover relationship of Hyperlipidemia and phospholipids, we carried out this study. Methods: We carried on the bioinformatics study on the relationship by Cytoscape software with Agilent Literature Search and ClusterONE plugins loaded. To search literatures of the relationship by Agilent Literature Search plugin and the network was generated and laid out in a new window. Loaded the above network into Cytoscape and new networks were generated by cluster analysis. Results: The network generated through Agilent Literature Search plugin includes 72 nodes and 95 edges. 14 clusters were generated by ClusterONE after cluster analysis, in which there are 7 clusters (p-value less than 0.05 ) were statistically significant, the node size is 3-7. The 7 predominant network pathway which could explain the relationship of Hyperlipidemia and phospholipids. Conclusion :The Cytoscape software with Agilent Literature Search and ClusterONE plugins is very powerful tool for discover potential pathways to explain Pathophysiology mechanism.
\end{abstract}

Keywords- Bioinformatics study, Hyperlipidemia, Phospholipids, Cytoscape, ClusterONE

\section{INTRODUCTION}

Hyperlipidemia involves abnormally elevated levels of any or all lipids and/or lipoproteins in the blood[1]. It is the most common form of dyslipidemia (which includes any abnormal lipid levels). Phospholipids are a class of lipids that are a major component of all cell membranes as they can form lipid bilayers. Some types of phospholipid can be split to produce products that function as second messengers in signal transduction [2]. Dietary fat intake is a risk factor for coronary heart disease and the modification of dietary habits is important for the prevention of cardiovascular disease (CVD) [3]. Hyperlipidemia is found to be associated with changes in fatty acid (FA) profiles. Concentration of essential n- 6 and $\mathrm{n}-3$ polyunsaturated fatty acid (PUFA) in cell membrane or in serum phospholipids are highly correlated with dietary intake [4].

Cytoscape is an open source software platform for visualizing molecular interaction networks and biological pathways and integrating these networks with annotations, gene expression profiles and other state data. Cytoscape core distribution provides a basic set of features for data integration, analysis, and visualization. Additional features are available as Apps (formerly called Plugins). Apps are available for network and molecular profiling analyses, new layouts, additional file format support, scripting, and connection with databases [5].

Agilent Literature Search Software is a meta-search tool for automatically querying multiple text-based search engines (both public and proprietary) in order to aid biologists faced with the daunting task of manually searching and extracting associations among genes/proteins of interest.Agilent Literature Search Software can be used in conjunction with Cytoscape, which provides a means of generating an overview network view of gene/protein associations [6].

The ClusterONE plugin finds overlapping protein complexes in a protein interaction network loaded into Cytoscape. ClusterONE-derived complexes for several yeast data sets showed better correspondence with reference complexes in the Munich Information Center for Protein Sequence (MIPS) catalog and complexes derived from the Saccharomyces Genome Database (SGD) than the results of seven popular methods. The results also showed a high extent of functional homogeneity [7]. 
In this study, we undertook bioinformatics study on relationship of Hyperlipidemia and phospholipids by Cytoscape software with Agilent Literature Search and ClusterONE plugins loaded, and found some important pathways to explain the relationship.

\section{METHODS}

Using Cytoscape version number 3.0, upload the Agilent Literature Search plugin. Go to Plugins->Manage Plugins. The Agilent Literature Search plugin will be in the Available for install folder. Bring up the Agilent Literature Search plugin via the Plugins menu. Type phospholipid into the Terms pane of Agilent Literature Search GUI. Click on Use Aliases in Search Controls. User context serves to limit the scope of the search, type Hyperlipidemia into the Context pane. Click on the blue Play button in the control panel. The literature search tool begin to exercise queries. When the query completes, a list of citations in the Query Results pane could be seen, then a network would be laid out in a New Network window.

Load the above network into Cytoscape. Click on Plugins/ClusterONE/Start to start ClusterONE. This will add a new panel called ClusterONE to the control panels on the left. Click on the Start button at the bottom of the panel. ClusterONE should detect the densely connected regions of your network and list them in a new panel on the Cytoscape result panel, which is usually on the right hand side of the window. Clicking on a cluster in the result panel will highlight the cluster in the network view. Double-clicking on a cluster will extract it as a new subnetwork. Right-clicking on a cluster will bring up a context menu with additional operations that you might find useful.

\section{RESULTS AND DISCUSSION}

The network generated through Agilent Literature Search plugin includes 72 nodes and 95 edges. Network circular layout is shown as Fig .1. ClusterONE layout after analysis is shown as Fig .2. Nodes are labeled with different colors and shapes in the latter figure, the network are more visualized.

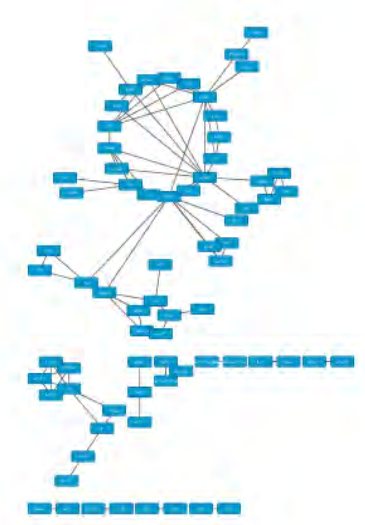

Figure 1. Circular network layout

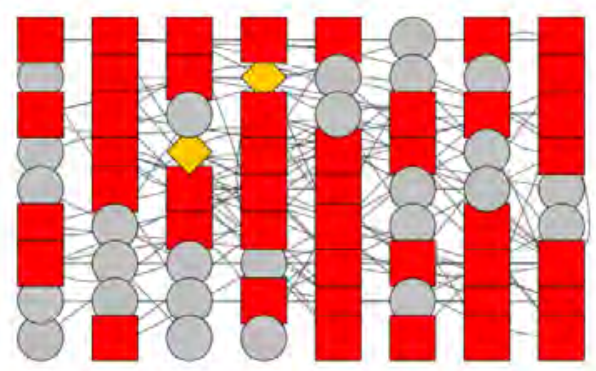

Figure 2. ClusterONE Network layout

14 clusters were generated by ClusterONE after cluster analysis, in which there are 7 clusters (p-value less than 0.05 ) were statistically significant, the node size is 3-7 (Table.1).

\section{TABLE I. Clusters Generated By ClusterONE}

\begin{tabular}{|c|c|c|c|c|c|c|c|}
\hline Cluster & Size & Density & $\begin{array}{c}\text { Internal } \\
\text { weight }\end{array}$ & $\begin{array}{c}\text { External } \\
\text { weight }\end{array}$ & Quality & $\mathrm{P}$-value & Members \\
\hline 1 & 5 & 1 & 10 & 2 & 0.003 & 0.002792 & $\begin{array}{r}\text { i16 s1pr1 s1pr3 mbtps1 } \\
\text { sphk1 }\end{array}$ \\
\hline 2 & 4 & 1 & 6 & 1 & 0.009 & 0.008853 & cc12 ets 1 ptk2b vcam1 \\
\hline 3 & 7 & 0.5238 & 11 & 7 & 0.018 & 0.017733 & $\begin{array}{c}\text { abca1 apoa1 scarb1 abcg1 lcat } \\
\text { pltp apob }\end{array}$ \\
\hline 4 & 3 & 1 & 3 & 0 & 0.023 & 0.023427 & $\begin{array}{c}\text { adipor } 2 \text { pdk4 pyruvate } \\
\text { dehydrogenase }\end{array}$ \\
\hline 5 & 3 & 0.6667 & 2 & 0 & 0.03 & 0.029673 & srebf1 scd1 scap \\
\hline 6 & 6 & 0.5333 & 8 & 6 & 0.034 & 0.034151 & $\begin{array}{c}\text { cd36 srebf2 acat1 fyn vav1 } \\
\text { sral }\end{array}$ \\
\hline 7 & 4 & 0.5 & 3 & 2 & 0.043 & 0.043179 & prkcc ptk2 src syk \\
\hline
\end{tabular}


The first cluster is shown as Fig .3. The network has 5 nodes and 10 edges. According to the network, SphK1/S1P signaling pathway is involved in the development of Hyperlipidemia. Fructose feeding induced hyperlipidemia, and activated SphK1/S1P signaling pathway characterized by the elevation of SphK1 activity, S1P production as well as SphK1, S1PR1 and S1PR3 protein levels, which in turn caused NF- $\kappa$ B signaling activation to produce IL-1 $\beta$, IL-6 and TNF- $\alpha$. Subsequently, hepatic insulin and leptin signaling impairment and lipid metabolic disorder were observed, resulting in liver lipid accumulation [8].

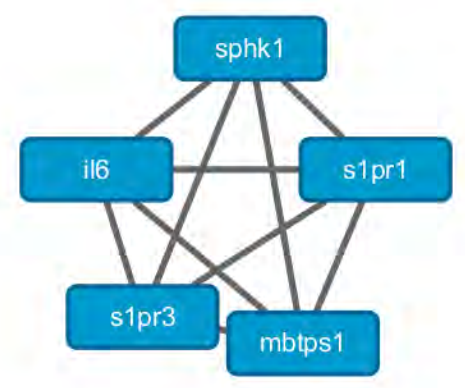

Figure 3. The first cluster network

The second cluster is shown as Fig .4. It has 4 nodes and 6 edges. The network is involved in proline-rich tyrosine kinase (PYK2)-mediated ROS generation pathway system. PYK2 is a key tyrosine kinase activated by high cholesterol exposure, which causes ROS-mediated $\mathrm{TNF} \alpha$ release and induces TNF $\alpha$-dependent expression of proinflammatory molecules [9].

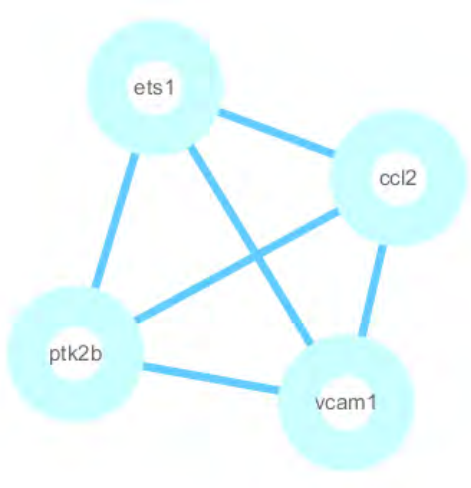

Figure 4. The second cluster network

The third cluster is shown as Fig .5. It has 4 nodes and 4 edges. The network is involved in classical MAPK signaling pathway, including ERK1/2, JNK, and p38. Labedipinedilol-A can suppress lysoPC-induced VSMCs death via reducing ROS production and anti-apoptosis. These protective effects are potentially mediated through the inhibition of $\mathrm{Ca}(2+)$ influx, and MAPK signaling, labedipinedilol-A may have a valuable role in the preventing atherosclerosis associated with hyperlipidemia[10].

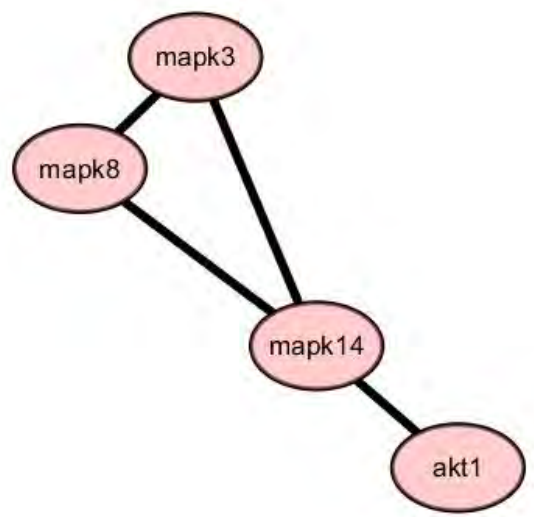

Figure 5. The third cluster network

The 4th cluster is shown as Fig .6. It has 3 nodes and 3 edges. Toll-like receptor (TLR)-2 and TLR-4, which are predominantly expressed on Activated monocytes and dendritic cells and mediate their activation, are essential for atherosclerosis development. Hyperlipidemia displayed a form of innate immune paralysis, including reduced pro- and anti-inflammatory cytokine release to external stimulus, which was related to the altered TLRNF- $\kappa \mathrm{B}$ signaling pathway and altered pro- and antiapoptotic processes in macrophages[11].

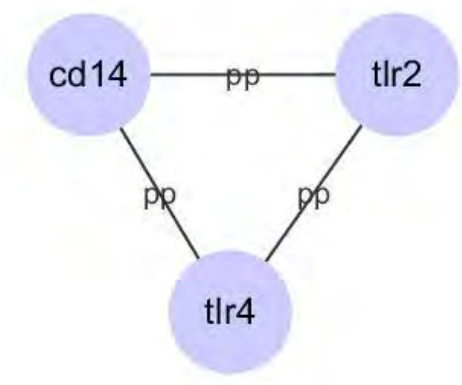

Figure 6. The fourth cluster network

The 5th cluster is shown as Fig .7. It has 6 nodes and 10 edges. Cholesterol efflux from macrophage foam cells, the initial step of reverse cholesterol transport (RCT) is the most relevant step with respect to atherosclerosis. The ATP-binding cassette (ABC) transporters ABCA1 and ABCG1 play crucial roles in the efflux of cellular cholesterol to HDL and its apolipoproteins. ABCA1 mutations can cause a severe HDL-deficiency syndrome characterized by cholesterol deposition in tissue macrophages and prevalent atherosclerosis. Cholesterol acyltranserase(LCAT), phospholipid transfer protein (PLTP),scavenger receptor class B type I (Sr-b1), and apolipoprotein AI (Apoa1) all play important roles in Hyperlipidemia pathogenesy [12]. 


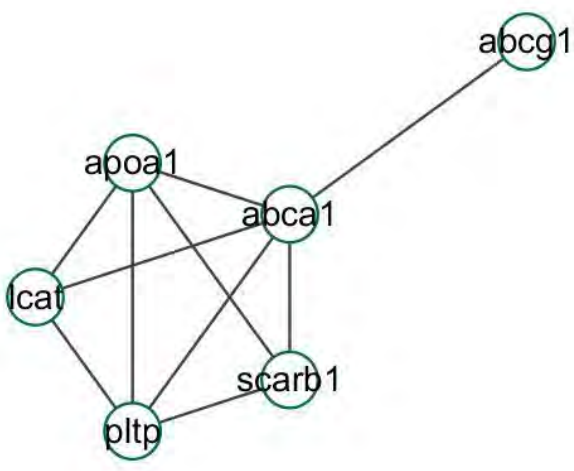

Figure 7. The fifth cluster network

\section{CONCLUSION}

We carried out the bioinformatics study on relationship of Hyperlipidemia and phospholipids by Cytoscape software with Agilent Literature Search and ClusterONE plugins loaded, and found 7 predominant network pathway which could explain the relationship of Hyperlipidemia and phospholipids. The Cytoscape software with Agilent Literature Search and ClusterONE plugins is very powerful tool for discover potential pathways to explain Pathophysiology mechanism. SphK1/S1P, PYK2-mediated ROS generation and MAPK signaling pathway may be play very important roles in Hyperlipidemia development and phospholipids induction.

\section{ACKNOWLEDGMENT}

The present study was supported by the Guangdong Natural Science Foundation of China (No. 10351022401000000).

\section{REFERENCES}

[1] Dorland's Medical Dictionary for Health Consumers, 2007,by Saunders, an imprint of Elsevier.

[2] S.-Y. Choi, J. Chang, B. Jiang, G.H. Seol, S.S. Min, J.S. Han, H.S Shin et al., "Multiple Receptors Coupled to Phospholipase C Gate Long-Term Depression in Visual Cortex". Journal of Neuroscience Vol. 25,2005 , p p.11433-43.

[3] Grundy S. M., Denke M. A.: Dietary influences on serum lipids and lipoproteins, J. Lipid Res. vol.31, 1990,pp.1149-1172.

[4] Ma J., Folsom A. R., Sharar E., Eckfeldt J. H. ,Plasma fatty acid composition as an indicator of habitual dietary fat intake in middleaged adults. Am. J. Clin. Nutr. vol.62, 1995,564-571.

[5] Information on http://www.agilent.ca/labs/research/litsearch.html.

[6] T. Nepusz, H. Yu, A. Paccanaro: Detecting overlapping protein complexes in protein-protein interaction networks, Nat Methods. vol. 9,2012, pp.471-472.

[7] J. Jahnel , P. Fickert, C. Langner, C. Högenauer, D. Silbert, J. Gumhold, A. Fuchsbichler, M. Trauner, Impact of experimental colitis on hepatobiliary transporter expression and bile duct injury in mice, Liver Int. vol. 29,2009, pp.1316-25.

[8] Wang X, Zhang DM, Gu TT, Ding XQ, Fan CY, Zhu Q, Shi YW, Hong Y, Kong LD. Morin reduces hepatic inflammation-associated lipid accumulation in high fructose-fed rats via inhibiting sphingosine kinase 1/sphingosine 1-phosphate signaling pathway. Biochem Pharmacol., vol.86,Dec.2013,pp1791-804,doi 10.1016/j.bcp.2013.10.005. Epub 2013 Oct 14

[9] Katsume A1, Okigaki M, Matsui A,et al. Early inflammatory reactions in atherosclerosis are induced by proline-rich tyrosine kinase/reactive oxygen species-mediated release of tumor necrosis factor-alpha and subsequent activation of the p21Cip1/Ets-1/p300 system,Arterioscler Thromb Vasc Bio,vol.31,2011,pp 1084-92. doi: 10.1161/ATVBAHA.110.221804

[10] Hsu JH1, Wu JR, Liou SF, Chen HM, Dai ZK, Chen IJ, Yeh JL. Labedipinedilol-A prevents lysophosphatidylcholine-induced vascular smooth muscle cell death through reducing reactive oxygen species production and anti-apoptosis. Atherosclerosis,vol. 217,2011,pp.379-86, doi: 10.1016/j.atherosclerosis.2011.04.022.

[11] Chen S1, Lin G, Lei L, You X, Wu C, Xu W, Huang M, Luo L, Wang Z, Li Y, Zhao X, Yan F. Hyperlipidemia modifies innate immune responses to lipopolysaccharide via the TLR-NF- $\mathrm{KB}$ signaling pathway. Inflammation,vol.36, 2013,pp.968-76,doi: 10.1007/s10753-013-9628-9.

[12] Ye D, Lammers B, Zhao Y, Meurs I, Van Berkel TJ, Van Eck M. ATP-binding cassette transporters A1 and G1, HDL metabolism, cholesterol efflux, and inflammation: important targets for the treatment of atherosclerosis, Curr Drug Targets, vol.5, 2011,pp.647-660. 